\title{
Algebroid Solutions of Second Order Complex Differential Equations
}

\author{
Lingyun Gao ${ }^{1}$ and Yue Wang ${ }^{2}$ \\ ${ }^{1}$ Department of Mathematics, Jinan University, Guangzhou, Guangdong 510632, China \\ ${ }^{2}$ School of Information, Renmin University of China, Beijing 100872, China \\ Correspondence should be addressed to Lingyun Gao; tgaoly@jnu.edu.cn
}

Received 28 November 2013; Accepted 17 December 2013; Published 2 January 2014

Academic Editor: Zong-Xuan Chen

Copyright (c) 2014 L. Gao and Y. Wang. This is an open access article distributed under the Creative Commons Attribution License, which permits unrestricted use, distribution, and reproduction in any medium, provided the original work is properly cited.

Using value distribution theory and maximum modulus principle, the problem of the algebroid solutions of second order algebraic differential equation is investigated. Examples show that our results are sharp.

\section{Introduction and Main Results}

We use the standard notations and results of the Nevanlinna theory of meromorphic or algebroid functions; see, for example, $[1,2]$.

In this paper we suppose that second order algebraic differential equation (3) admit at least one nonconstant $\nu$ valued algebroid solution $w(z)$ in the complex plane. We denote by $E$ a subset of $[0, \infty)$ for which $m(E)<\infty$ and by $K$ a positive constant, where $m(E)$ denotes the linear measure of $E$. $E$ or $K$ does not always mean the same one when they appear in the following.

Let $a_{j k}\left(j=0,1, \ldots, n ; k=0,1, \ldots, q_{j}\right)$ be entire functions without common zeroes such that $a_{0 q_{0}} \neq 0$. We put

$$
\begin{aligned}
& Q_{j}(z, w)=\sum_{k=0}^{q_{j}} a_{j k} w^{k}, \quad q_{j}=\operatorname{deg}_{w}^{Q_{j}} ; \\
& p=\max \left\{q_{j}+j: j=0,1, \ldots, n-1\right\} .
\end{aligned}
$$

Some authors had investigated the problem of the existence of algebroid solutions of complex differential equations, and they obtained many results ([2-10], etc.).

In 1989, Toda [4] considered the existence of algebroid solutions of algebraic differential equation of the form

$$
\sum_{j=0}^{n} Q_{j}(z, w)\left(w^{\prime}\right)^{j}=0 .
$$

He obtained the following.
Theorem A (see [4]). Let $w(z)$ be a nonconstant $v$-valued algebroid solution of the above differential equation and all $a_{j k}$ are polynomials. If $p<n+q_{n}$, then $w(z)$ is algebraic.

The purpose of this paper is to investigate algebroid solutions of the following second order differential equation in the complex plane with the aid of the Nevanlinna theory and maximum modulus principle of meromorphic or algebroid functions:

$$
\sum_{j=0}^{n} Q_{j}(z, w)\left(w^{\prime \prime}\right)^{j}=0,
$$

where $Q_{j}(z, w)=\sum_{k=0}^{q_{j}} a_{j k} w^{k}, j=0,1,2, \ldots, n$.

We will prove the following two results.

Theorem 1. Let $w(z)$ be a nonconstant $v$-valued algebroid solution of differential equation (3) and all $a_{j k}$ are polynomials. If $p \leq q_{n}$, then $w(z)$ is algebraic, $p=\max \left\{q_{j}: j=0,1, \ldots, n-\right.$ $1\}$.

Theorem 2. Let $w(z)$ be a nonconstant $v$-valued algebroid solution of differential equation (3) and the orders of all $a_{j k}$ are finite. If $q_{0}>\max _{1 \leq j \leq n-1}\left\{q_{j}+j\right\}$, then the following statements are equivalent:
(a) $\delta(\infty, w)>0$;
(b) $q_{0}=q_{n}+n$;
(c) $\infty$ is a Picard exceptional value of $w(z)$. 


\section{Some Lemmas}

Lemma 3 (see [2]). Suppose that $w(z), a_{i}(z),(i=1,2, \ldots, p)$ are meromorphic functions, and $a_{p}(z) \neq 0$. Then one has

$$
m\left(r, \sum_{i=1}^{p} a_{i}(z) w^{i}\right) \leq p m(r, w)+\sum_{i=1}^{p} m\left(r, a_{i}(z)\right)+O(1) .
$$

Examining proof of Lemma 4.5 presented in [2, pp. 192193], we can verify Lemma 4.

Lemma 4. Let $w(z)$ be a transcendental algebroid function such that $w(z)$ has only finite number of poles, and let $w(z)$, $w^{\prime}(z)$, and $w^{\prime \prime}(z)$ have no poles in $|z|>r_{0}$. Then, for some constants $C_{i}>0, i=1,2,3$, and $r \geq r_{1} \geq r_{0}$ it holds:

$$
M(r, w) \leq C_{1}+C_{2} r+C_{3} r^{2} M\left(r, w^{\prime \prime}\right),
$$

where $M(r, w)=\max _{|z|=r}\{|w(z)|\}$.

Lemma 5 (see [11]). The absolute values of roots of equation

$$
z^{n}+a_{1} z^{n-1}+\cdots+a_{n}=0
$$

are bounded by

$$
\max \left\{n\left|a_{1}\right|,\left(n\left|a_{2}\right|\right)^{1 / 2}, \ldots,\left(n\left|a_{n}\right|\right)^{1 / n}\right\} .
$$

Lemma 6. Let $w(z)$ be a nonconstant $v$-valued algebroid solution of the differential equation (3) and let $a_{j k}$ be a polynomial. If $p<n+q_{n}$, then

$$
\begin{aligned}
\min \{n & \left., q_{n}-p\right\} \log ^{+} M(r, w) \\
& +\max \left\{0, q_{n}-p-n\right\} \log ^{+} M(r, w) \\
\leq & +O(\log r)+O(1), \quad(r \notin E),
\end{aligned}
$$

where $M(r, w)=\max _{|z|=r}\{|w(z)|\}, K$ is a positive constant.

Proof. We first prove that the poles of $w$ are contained in the zeroes of $\left\{a_{n q_{n}}(z)\right\}$.

Suppose that $z_{0}$ is a pole of $w$ of order $\tau$ and $z_{0}$ is not the zeroes of $\left\{a_{n q_{n}}(z)\right\}$. Then

$$
\begin{gathered}
w(z)=\left(z-z_{0}\right)^{-\tau / \lambda} w_{1}(z), \quad w_{1}\left(z_{0}\right) \neq 0, \infty, \\
w^{\prime \prime}(z)=\left(z-z_{0}\right)^{-(\tau+2 \lambda) / \lambda} w_{2}(z), \quad w_{2}\left(z_{0}\right) \neq 0, \infty .
\end{gathered}
$$

We rewrite differential equation (3) as follows:

$$
a_{n q_{n}}\left(w^{\prime \prime}\right)^{n}=\sum_{j=0}^{n-1} Q_{j}(z, w)\left(w^{\prime \prime}\right)^{j}
$$

It follows from (10) that

$$
q_{n} \tau+n(\tau+2 \lambda) \leq p \tau+(n-1)(\tau+2 \lambda) .
$$

Noting that $p \leq q_{n}$, we have

$$
\tau+2 \lambda<0
$$

This is a contradiction.

This shows that the poles of $w$ are contained in the zeroes of $\left\{a_{n q_{n}}(z)\right\}$.

We rewrite differential equation (3) as follows:

$$
\begin{gathered}
\sum_{j=0}^{n} Q_{j}(z, w) Q_{n}(z, w)^{n-j-1}\left(Q_{n} w^{\prime \prime}\right)^{j}=0 . \\
M\left(r, Q_{1} w^{\prime \prime}\right) \geq M(r, w)^{q_{n}}+M\left(r, w^{\prime \prime}\right) \\
-\sum_{k=0}^{q_{n}-1} M\left(r, a_{n k}\right) M(r, w)^{k} \\
\geq M(r, w)^{q_{n}}+\frac{M(r, w)-C_{1}-C_{2} r}{C_{3} r^{2}} \\
-\sum_{k=0}^{q_{n}-1} M\left(r, a_{n k}\right) M(r, w)^{k} .
\end{gathered}
$$

For $j=0,1, \ldots, n-1$, we have

$$
\left|Q_{j}\left(z_{r}, w\right) Q_{n}\left(z_{r}, w\right)^{n-j-1}\right| \leq K M(r, w)^{q_{j}+q_{n}(n-j-1)} .
$$

Applying Lemma 5 to (13) at $z=z_{r}$,

$$
M\left(r, Q_{1} w^{\prime \prime}\right) \leq K M(r, w)^{\max \left\{h_{j}: j=0,1, \ldots, n-1\right\}},
$$

where $h_{j}=\left(q_{j}+q_{n}(n-j-1)\right) /(n-j)$.

From (14) and (15), we have

$$
\begin{aligned}
M(r, w)^{q_{n}} & \leq K\left\{M(r, w)^{q_{n}-1}+r^{2} M(r, w)^{\max \left\{h_{j}: j=0,1, \ldots, n-1\right\}}\right\} \\
& \leq K\left\{M(r, w)^{q_{n}-1}+r^{2} M(r, w)^{q_{n}+\left(\left(p-q_{n}\right) / n\right)}\right\} .
\end{aligned}
$$

Note that

$$
\begin{aligned}
h_{j} & =\frac{q_{j}+q_{n}(n-j-1)}{n-j}<\frac{p+q_{n}(n-j-1)-j}{n-j} \\
& =q_{n}+\frac{p-q_{n}}{n}+\frac{j\left(p-q_{n}-n\right)}{n(n-j)} \\
& \leq q_{n}+\frac{p-q_{n}}{n}, \quad j=0,1, \ldots, n-1 .
\end{aligned}
$$

Dividing the inequality (17) by $M(r, w)^{\max \left\{q_{n}-1, q_{n}+\left(\left(q_{n}-p\right) / n\right)\right\}}$, we obtain, for $r \notin E$,

$$
M(r, w)^{\min \left\{1,\left(q_{n}-p\right) / n\right\}} \leq K_{4}\left\{1+\frac{r^{2}}{M(r, w)^{\max \left\{0,\left(q_{n}-p-n\right) / n\right\}}}\right\},
$$

which reduces to our inequality by calculating $\log ^{+}$of the both sides:

$$
\begin{aligned}
& \min \left\{n, q_{n}-p\right\} \log ^{+} M(r, w) \\
& \leq-\max \left\{0, q_{n}-p-n\right\} \log ^{+} M(r, w)+O(\log r)+O(1) .
\end{aligned}
$$

Lemma 6 is complete. 


\section{Proof of Theorem 1}

First, we consider $N(r, w)$.

Let $z_{0}$ be a pole of $w$ of $\tau$. Let $t$ be the order of zero of $a_{n q_{n}}(z)$ at $z_{0}$.

(i) When the order of the pole of $Q_{n}(w)\left(w^{\prime \prime}\right)^{n}$ is not equal to that of other terms of the left-hand side of (10) at $z_{0}$, we get

$$
q_{n} \tau+n(\tau+2 \lambda)-t \lambda \leq p \tau
$$

that is,

$$
\tau \leq \frac{(t-2 n) \lambda}{q_{n}+n-p} .
$$

(ii) When the order of pole of $Q_{n}(w)\left(w^{\prime \prime}\right)^{n}$ is equal to that of some term $Q_{k}(w)\left(w^{\prime \prime}\right)^{n}$ of the left-hand side of (10) at $z_{0}$, we get

$$
q_{n} \tau+n(\tau+2 \lambda)-t \lambda \leq p \tau+n(\tau+2 \lambda)
$$

that is,

$$
\tau \leq \frac{t \lambda}{q_{n}-p} .
$$

Combining cases (i) and (ii), we obtain

$$
N(r, w) \leq K_{8} N\left(r, \frac{1}{a_{n q_{n}}}\right),
$$

where $K_{8}$ is a positive constant.

Secondly, by Lemma 6, we obtain

$$
\begin{aligned}
\min & \left\{n, q_{n}-p\right\} m(r, w) \\
\leq K & {\left[\sum_{i=0}^{p} m\left(r, a_{i}\right)+\sum_{k=0}^{q_{n}-1} m\left(r, b_{k}\right)\right]+O(\log r), \quad(r \notin E) . }
\end{aligned}
$$

Combining the inequalities (25) and (26), we have

$$
T(r, w)=O(\log r), \quad(r \notin E),
$$

which shows that $w$ is an algebraic solution of (3).

This completes the proof of Theorem 1 .

\section{Proof of Theorem 2}

(i) (a) $\Rightarrow(b)$. Suppose that $\delta(\infty, w)>0$. If $q_{0}>q_{n}+n$, then we have by (3)

$$
w^{q_{0}}=-\frac{1}{a_{0 q_{0}}}\left\{\sum_{j=1}^{n} Q_{j}(w) w^{j}\left(\frac{w^{\prime \prime}}{w}\right)^{j}-\sum_{k=0}^{q_{0}-1} a_{0 k} w^{k}\right\} .
$$

Applying Lemma 3 to (28),

$$
\begin{aligned}
q_{0} m(r, w) \leq & \left(q_{0}-1\right) m(r, w)+\sum_{j, k} m\left(r, a_{j k}\right) \\
& +K m\left(r, \frac{w^{\prime \prime}}{w}\right)+m\left(r, \frac{1}{a_{0 q_{0}}}\right)+O(1) .
\end{aligned}
$$

Since $w(z)$ is admissible solution, we have

$$
m(r, w)=S(r, w),
$$

so that

$$
\delta(w, \infty)=0 .
$$

This is a contradiction. Thus, $q_{0} \leq q_{n}+n$.

If $q_{0}<q_{n}+n$, by Theorem $1, w(z)$ is nonadmissible. Thus,

$$
q_{0}=q_{n}+n
$$

(ii) (b) $\Rightarrow$ (c). Let $q_{0}=q_{n}+n$. Then, similar to the proof of Lemma 6 , we obtain that the poles of $w(z)$ are contained in the set of $a_{n q_{n}}$ and $\infty$ is a Picard exceptional value of $w(z)$.

(iii) (c) $\Rightarrow(a)$. Let $\infty$ be a Picard exceptional value of $w(z)$. Then $\delta(w, \infty)=1$.

\section{Some Examples}

Example 1. The differential equation

$$
v^{2} w^{2 v-1} w^{\prime \prime}-\left[\left((2 v+1) w^{4 v}+2 w^{2 v}-v+1\right)\right]=0
$$

has a transcendental algebroid solution $w(z)=(\tan z)^{1 / \nu}$. In this case

$$
p=q_{0}=4 \nu>1+q_{1}=2 \nu-1+1=2 \nu .
$$

Remark 7. Example 1 shows that the condition in Theorem 1 is sharp.

Example 2. Transcendental algebroid function $w(z)=$ $(\sin z)^{1 / 2}$ is a 2 -valued solution of the following differential equation:

$$
16 w^{6}\left(w^{\prime \prime}\right)^{2}+2 w^{5} w^{\prime \prime}-\left(w^{8}-\frac{1}{2} w^{6}+2 w^{4}-\frac{1}{2} w^{2}+1\right)=0 .
$$

In this case

$$
q_{0}=8, \quad n=2, \quad q_{1}=5, \quad q_{2}=6 .
$$

By Theorem 2, for transcendental algebroid function $w(z)=$ $(\sin z)^{1 / 2}, \infty$ is a Picard exceptional value.

Remark 8. Example 2 shows that the result in Theorem 2 holds.

\section{Conflict of Interests}

The authors declare that there is no conflict of interests regarding the publication of this paper.

\section{Acknowledgments}

This project is project Supported by National Natural Science Foundation (10471065) of China and NSF of Guangdong Province (04010474). 


\section{References}

[1] H. Yi and C. C. Yang, Theory of the Uniqueness of Meromorphic Functions, Science Press, Beijing, China, 1995 (Chinese).

[2] Y. Z. He and X. Z. Xiao, Algebroid Functions and Ordinary Differential Equations, Science Press, Beijing, China, 1988.

[3] Y. Z. He and X. Z. Xiao, "Admissible solutions and ordinary differential equations," Contemporary Mathematics, vol. 25, pp. 51-61, 1983.

[4] N. Toda, "On algebroid solutions of some algebraic differential equations in the complex plane," Japan Academy A, vol. 65, no. 4, pp. 94-97, 1989.

[5] T. Chen, "One class of ordinary differential equations which possess algebroid solutions in the complex domain," Chinese Quarterly Journal of Mathematics, vol. 6, no. 4, pp. 45-51, 1991.

[6] K. Katajamäki, "Value distribution of certain differential polynomials of algebroid functions," Archiv der Mathematik, vol. 67, no. 5, pp. 422-429, 1996.

[7] L. Gao, "Some results on admissible algebroid solutions of complex differential equations," Indian Journal of Pure and Applied Mathematics, vol. 32, no. 7, pp. 1041-1050, 2001.

[8] L.-Y. Gao, "On some generalized higher-order algebraic differential equations with admissible algebroid solutions," Indian Journal of Mathematics, vol. 43, no. 2, pp. 163-175, 2001.

[9] L. Gao, "On the growth of solutions of higher-order algebraic differential equations," Acta Mathematica Scientia B, vol. 22, no. 4, pp. 459-465, 2002.

[10] L. Y. Gao, "The growth of single-valued meromorphic solutions and finite branch solutions," Journal of Systems Science and Mathematical Sciences, vol. 24, no. 3, pp. 303-310, 2004.

[11] T. Takagi, Lecture on Algebra, Kyoritsu, Tokyo, Japan, 1957 (Japanese). 


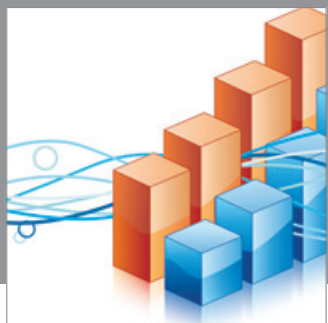

Advances in

Operations Research

mansans

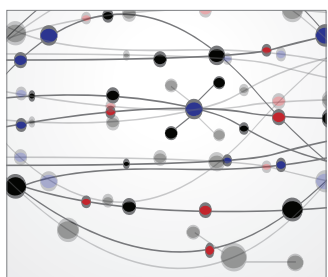

The Scientific World Journal
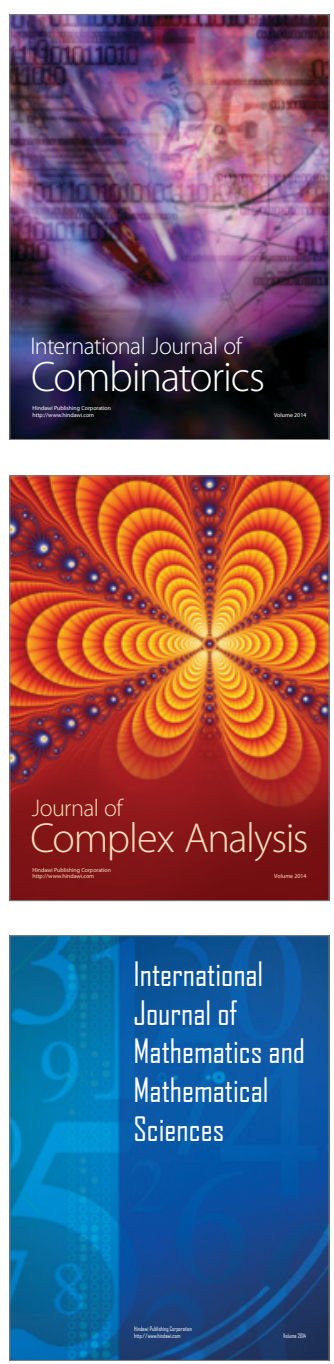
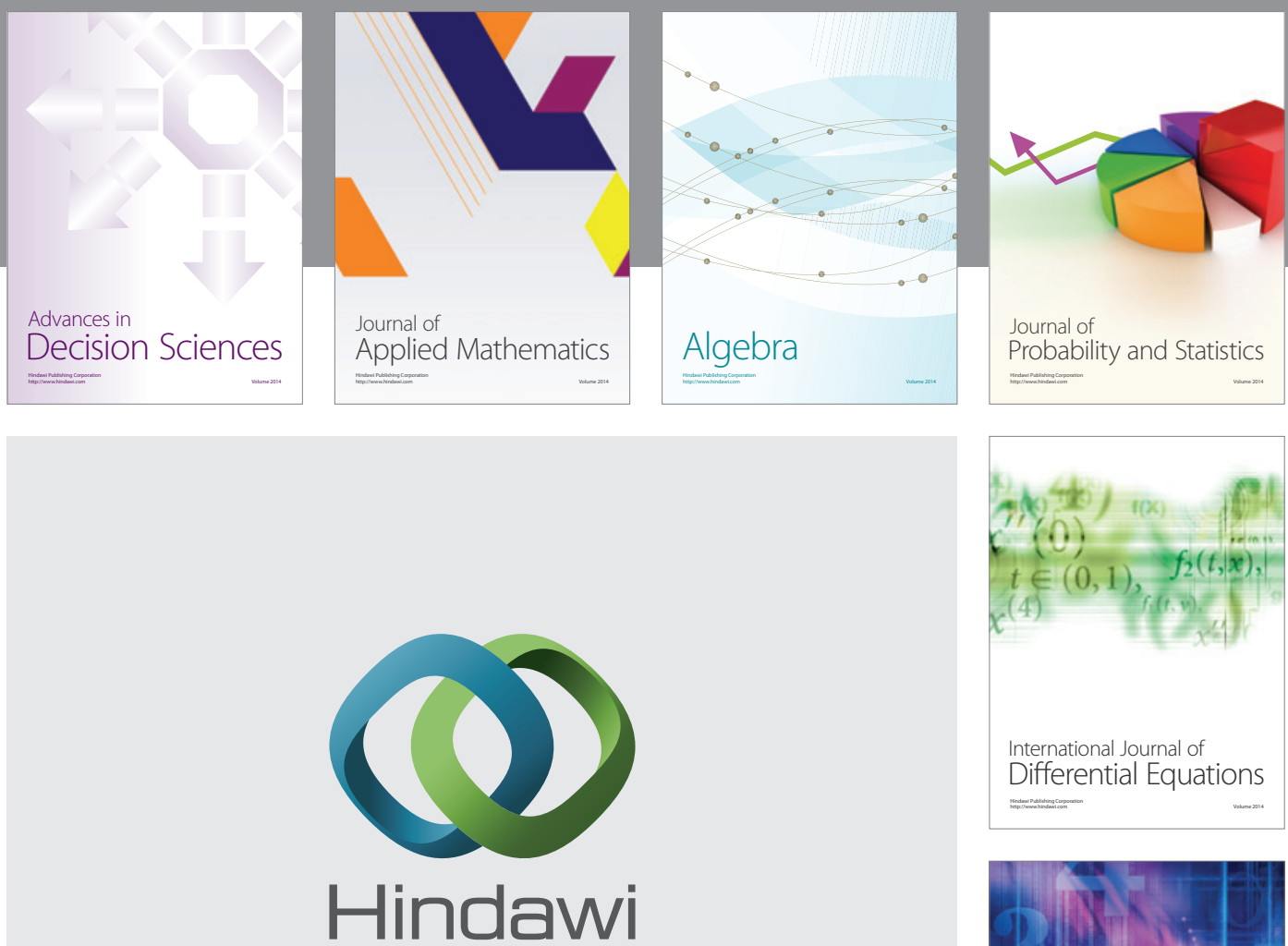

Submit your manuscripts at http://www.hindawi.com
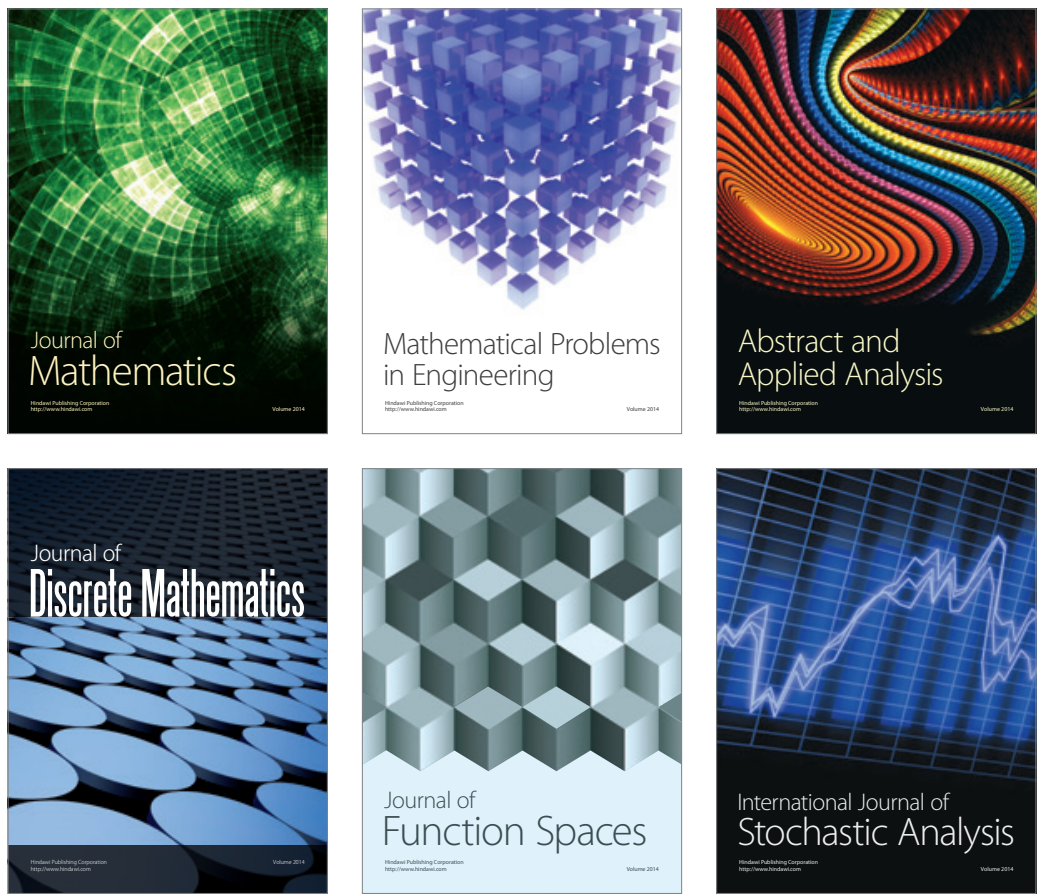

Journal of

Function Spaces

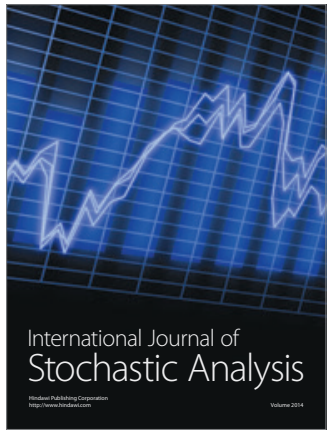

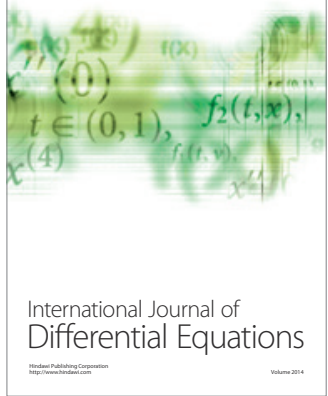
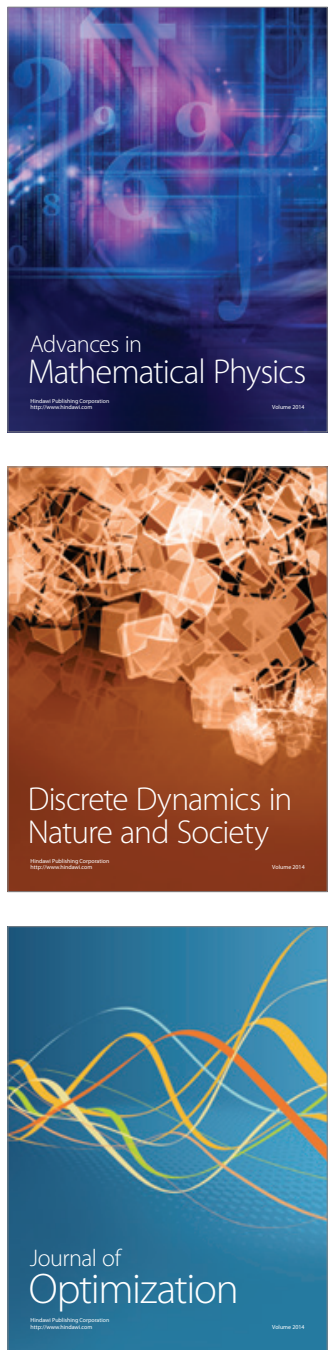